\title{
Mind in emergency: Needs, criteria, priorities and methods
}

\author{
Fabio Sbattella \\ Emergency Psychology Research Unit, Catholic University of the Sacred Heart, Milan, Italy
}

\section{Introduction}

This editorial asks many questions and tries to offer some partial answers. Two main questions are: how does the mind work in emergency conditions? What care must medical assistance have to involve the mind effectively in an emergency situation?

To answer the first question, we need to investigate some aspects in more detail. First of all: what do we mean by mind? And then: how do we define an emergency condition?

The second question is related to behaviors and procedures to be used in the field. To find and use effective and successful methodologies for rescue actions, we need again to be more specific. First of all: what does the mind need in emergency conditions to function well? And then: what mind are we talking about? The minds of victims, rescuers, witnesses, survivors or relatives of the victims? If we consider all these minds as variables that intervene in the context of disaster management, we will have to ask ourselves: do they have different needs? How do they interact with each other? Only at this point, will we be able to decide which criteria to adopt in making clinical and operative choices, which priorities to establish and how to integrate the various interventions into successive phases. ${ }^{1}$

All these questions constitute the heart of research in emergency psychology, a field of study and intervention apparently young and rapidly growing, but with ancient roots in the psychology of war, trauma and the first disasters of the twentieth century. ${ }^{2}$ Some ideas will be offered in this editorial, in order to summarize this complex research field. More practical examples and further discussion will come from the articles published in this issue of the Emergency Care Journal and possibly in future ones that the magazine might be able to host.

Correspondence: Fabio Sbattella, Emergency Psychology Research Unit, Catholic University of the Sacred Heart, Largo Gemelli 1, 20123 Milan, Italy.

Tel. +39.02.7234.2284 - Fax: +39.02.7234.2280.

E-mail: fabio.sbattella@unicatt.it

Key words: Emergency; Disrupted mind; Psychological interventions.

Received for publication: 25 October 2019

Accepted for publication: 28 October 2019

This work is licensed under a Creative Commons Attribution 4.0 License (by-nc 4.0).

๑C Copyright: the Author(s), 2019

Licensee PAGEPress, Italy

Emergency Care Journal 2019; 15:8641

doi:10.4081/ecj.2019.8641

\section{The mind as a relational device}

A common misconception is to think that the mind (psyche) coincides with the brain. Indeed, they are two realities intimately connected to each other, but precisely distinguished from an epistemological and practical point of view. The mind is in fact the set of psychic functions that regulate the balance between a living organism and its environment. In the case of human beings, these functions include the processes of attention, perception, memory, emotion, reasoning, learning, communication. The brain is instead a biological entity, inseparable from and integrated with the rest of the nervous system and with the whole body. The relationships between mind and brain (or rather, with the body as a whole) are being extensively studied, but it is clear to many that the mind is not reducible to the brain. The mind emerges from the general functioning of the body and from an inseparable interaction with the environment. ${ }^{3}$ The evidence that supports this position emerges clearly during accidents, disasters and catastrophes. The destruction of objects or territories and the loss of people profoundly disrupt the minds individuals, groups and communities. Much of the long-term memory that allows the functioning of the mind is in fact deposited in significant objects and persons. When disrupted minds cannot access this information, they have difficulties in thinking, emotional adjustment, organization and behavioral guidance. Using a metaphor, we could compare the brain with a hardware that works exclusively thanks to the stored information and many software (the mind) that largely reside in the cloud. Without a network access, hardware designed to run in the cloud will be very inefficient. Similarly, without the ability to communicate or connect with people and objects that are meaningful to them, human minds will enter inefficient loops, showing discomfort and maladaptive or dysfunctional behaviors. ${ }^{4}$

\section{Emergency}

An emergency can be defined as a situation that is created by a rapid, sudden and devastating environmental change. In particular, we want to emphasize that an emergency is not an objective fact, but an organizational condition. The objective facts are rapid, sudden and devastating changes (for example, a volcanic eruption, an earthquake, an explosion, a terrorist attack, a traffic accident). The emergency is instead the set of ways in which these events are elaborated by the mind at an organizational, group and individual level. The emergency condition has some specific characteristics: it is an interactive context characterized by the perception of a threat, by the request for decisions and quick actions, by the perception of a disproportion between emerging needs and available resources and by congruent emotions.

This definition offers tools to understand the needs of a mind in emergency conditions. The perception of an emergency grows when people interact with each other over evaluating the signs of a serious and devastating danger; when they ask for hasty decisions and say that there is no time to think before acting; when they 
under evaluate the resources needed to cope with emerging needs; when they expand the burden of dramatic questions that must be answered immediately; when they do not modulate the emotions that naturally accompany these processes.

To function well in the face of a critical environmental change, to manage and exit the state of emergency, the mind needs instead to actively interact in order to: clarify the nature, gravity and proximity of the imminent threats; fielding decision-making processes functional to the chaotic context (as happens for example with heuristic decision strategies); gain time (for example to breathe and oxygenate the hardware that risks overheating); have additional resources (information, connections to other minds, solutions); to maintain empowerment (the feeling of being able to do something good while being powerless); dispose of actions or instruments of emotional regulation; reconnect with known people, personal objects (for example the smartphone), known places.

How is it possible to do all this in complex and chaotic contexts? Field experiences show that it is possible to work by setting some priorities and criteria. First of all, it is necessary to give priority to the collective and group mind (climate in the rescue team; family reunification, etc.): in this way the connections that the mind needs are facilitated. Secondly, it is essential to prepare (before the devastating changes arrive) the organizational mind (people, procedures, values) for the inclusion of the psychological dimensions in all phases of the rescue. Only with careful preparation before emergencies will it be possible to manage all the needs during and after the most dramatic moments.

Thirdly, using precise intervention techniques functional to precarious and chaotic contexts and acting in an integrated manner with all the forces in the field.

Finally, working to elaborate experiences, that is, to integrate and connect memories (episodic and sensorial) and things learned in the general system of the Self. If this does not happen, in the long term the disconnections are transformed into those fractures that clinical psychology has classified as traumatic syndromes. ${ }^{5}$

The experiences presented in this issue of the Emergency Care Journal document some good practices implemented during recent interventions in Italy. Several teams took charge of the psychological needs of rescuers, victims, family members and spectators during and after dramatic events of different sizes. These Case Histories testify to the wealth of experiences and methodologies already present in the sector in Italy. They also clarify the need for increasingly integrated (not only juxtaposed) work between those who deal with the needs of the body and those who deal with mind care. ${ }^{6}$

Hardware and software are made for each other: nothing works if they are not designed together.

\section{References}

1. Schnyder U. Early intervention in emergencies. Oxford: Oxford University Press; 2003.

2. Norris FH, Friedman MJ, Watson PJ, et al. 60,000 disaster victims speak: Part I. An empirical review of the empirical literature, 1981-2001. Psychiatry 2002;65:207-39.

3. Bateson G. Mind and nature: a necessary unit. New York, USA: Dutton; 1979.

4. Ardino V. Post-traumatic syndromes in childhood and adolescence: a handbook of research and practice. John Wiley \& Sons; 2011.

5. Bedin AG, Castelli C, Sbattella F. CAPS-CA Clinician-administered PTSD scale for children and adolescents. Florence, Italy: Giunti OS; 2013.

6. Sbattella F, Tettamanzi M, Iacchetti F. Basic therapeutic actions. Nuove Tend Psicol 2005;3:399-429. 